Results: In total, 312 consecutive AS patients, 34\% female, were included with a mean follow-up of 18.9 months. Most patients (172, 55\%) showed significant improvement after start of TNFi of whom 86 patients $(28 \%)$ had a clinically important (ASDAS decrease $>1.1$ ) and $86(26.9 \%)$ a major clinical improvement (ASDAS decrease $>2.2$ ). BMI was significantly correlated with age at diagnosis $(p=0.016 ; 95 \% \mathrm{Cl}: 0.07-0.65)$ : an increase of BMI with three points delayed the AS diagnosis with one year. At baseline, smoking and gender were not correlated with the ASDAS, but BASDAI and BASMI were both inversely related to BMI. Male gender was significantly associated with a higher chance at clinical response (improvement of the BASDAI with $50 \%$ or a 2 point decrease) to TNFi ( $\mathrm{p}=0.041$ ). At one year follow-up the clinical improvement of males versus females was respectively $62 \%$ vs. $43 \%$ and at two year follow-up $59 \%$ vs. $46 \%$. Males also showed a significantly higher ASDAS improvement after one year of follow-up compared to females $(p=0.015)$.

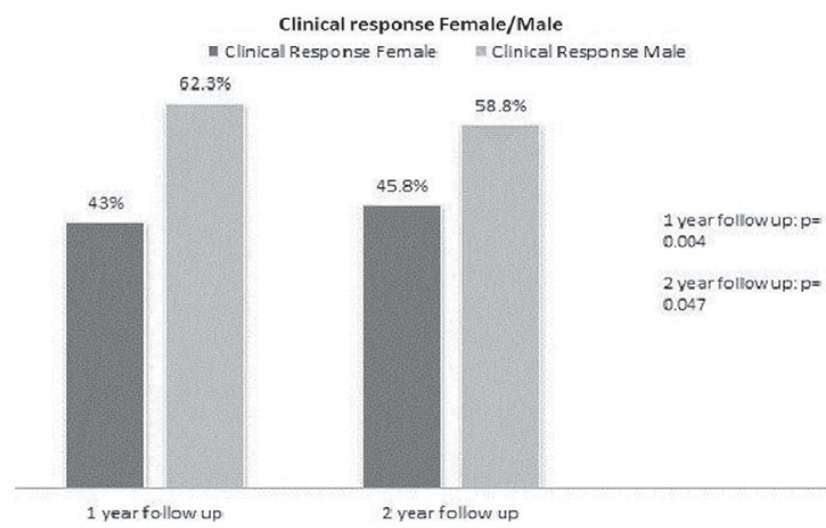

Conclusions: Significantly less females had a clinical response compared to males after one and two years of TNFi treatment. A higher BMI not only prolonged the time to AS diagnosis up to one year, but also negatively influenced the BASDAI and BASMI scores. Female gender and high body weight should be taken into consideration when the efficacy of TNFi is assessed, by stratifying for these factors in the analysis.

Disclosure of Interest: None declared

DOI: 10.1136/annrheumdis-2017-eular.1290

\section{THU0392 DO WE REALLY NEED DMARDS ADDITION TO ANKYLOSISNG SPONDYLITIS PATIENTS TREATED WITH TUMOR NECROSIS FACTOR ALPHA INHIBITORS?}

T. Reitblat ${ }^{1}$, O. Cohen ${ }^{1}$, D. Aharoni ${ }^{2}$, E. Hadrian ${ }^{2}$, G. Reifman ${ }^{1}$, Y. Braun-Moscovici ${ }^{3}$, A. Balbir-Gurman ${ }^{3} \cdot{ }^{1}$ Department of Rheumatology; ${ }^{2}$ Laboratory Service, Barzilai Mc, Ashkelon; ${ }^{3} B$. Shine Rheumatology Unit, Rambam Health Care Campus and Rappaport Faculty of Medicine, Technion, Heifa, Israel

Background: The management of systemic inflammatory diseases, such as Rheumatoid arthritis (RA) and Ankylosing spondylitis (AS) has been revolutionized with the introduction of tumor necrosis factor alpha inhibitors (TNF-I). Significant reduction in disease activity and achievment of remission resulted in halting of joint damage and improved quality of life. Unfortunately, approximatly $15-30 \%$ of patients fail to reach desirable improvement or lose drug effectiveness with time. It may be explained by immunogenicity and production of human anti chimeric antibodies (HACA). Presence of HACA against TNF-I have been associated with low levels of the drug and lose of therapeutic response. The prevalence of HACA in RA is estimated between 20-40\%, and in AS between 25-64\%. The difference in the pathogenesis of RA and AS as well as diverce approach in using disease modifying anti-rheumatic drugs (DMARDs) may influence HACA production and TNF-I levels in these conditions.

Objectives: To compare the incidence of HACA and Infliximab, Etanercept, Adalimumab levels in patients with RA and AS with respect to concomitant DMARDs.

Methods: Patients' with RA and AS data treated with TNF-I for at least 3 months in whom tests for HACA and Infliximab, Etanercept, Adalimumab levels were available were extracted from patients' files. Data included: demographics, concomitant treatment, and disease activity scores (BASDAI for AS and DAS-28 for RA). Serum for assessment of drugs level (ELISA) and HACA (ADAb;Promonitor, Bridging, ELISA) was obtained before the next drug administration. Univariate comparison was done using Student $t$ test for the continuous variables and $\mathrm{Chi}$ square test for the categorical variables $(\mathrm{P}<.05$ was considered significant) Results: Data on 53 patients with AS (mean age $47.9 \pm 11.9$ years, 41.5 . \% female) and 29 patients with RA (mean age $54.8 \pm 8.1$ years, 75, 9\% female) were available: 22 RA patients were treated with Methotrexate and 7 with other DMARDs; no one AS patient was treated with concomitant DMARDs. High level of HACA was found in $22.6 \%$ AS patients and in $34.5 \%$ RA patients ( $p>0.05$ ); $86.8 \%$ patients with AS reached theurapethic level of drug compared to $69 \%$ RA patients $(p=0.027)$. Drugs levels were similar in AS and RA patients (Table). Low disease activity (DAS28-CRP <3.2) was registered in 62.1\% RA patients and in $81.4 \%$ AS patients (BASDAI <4).

Conclusions: Despite significant difference in the use of concomitant DMARDs, patients with AS and RA had similar prevalence of HACA. Moreover, higher proportion of patients in AS group reached therapeutic level of TNF-I. The data supports the hypothesis, that immune responce to TNF-I may be different in AS and RA and, therefore, the addition of DMARDs to prevent development of HACA in patients with AS may be unnecessary.

Disclosure of Interest: None declared

DOI: 10.1136/annrheumdis-2017-eular.2584

\section{THU0393 SECUKINUMAB PROVIDES SUSTAINED REDUCTION IN FATIGUE IN PATIENTS WITH ANKYLOSING SPONDYLITIS THROUGH 3 YEARS: LONG-TERM RESULTS OF TWO RANDOMISED DOUBLE-BLIND PLACEBO-CONTROLLED PHASE 3 STUDIES}

T.K. Kvien ${ }^{1}$, A. Deodhar ${ }^{2}$, L. Gossec ${ }^{3}$, P.G. Conaghan ${ }^{4}$, V. Strand ${ }^{5}$, M. Østergaard ${ }^{6}$, N. Williams ${ }^{7}$, B. Porter ${ }^{8}$, K. Gandhi ${ }^{8}$, S. Jugl ${ }^{9}$.

${ }^{1}$ Diakonhjemmet Hospital, Oslo, Norway; ${ }^{2}$ Oregon Health \& Science University, Portland, Oregon, United States; ${ }^{3}$ UPMC University Paris 06, Paris, France; ${ }^{4}$ University of Leeds, Leeds, United Kingdom; ${ }^{5}$ Stanford University School of Medicine, Palo Alto, CA, United States; ${ }^{6}$ Copenhagen Center for Arthritis Research (COPECARE), Glostrup, Denmark; ${ }^{7}$ RTI Health Solutions, Durham, NC; ${ }^{8}$ Novartis Pharmaceuticals Corporation, East Hanover, NJ, United States; ${ }^{9}$ Novartis Pharma AG, Basel, Switzerland

Background: In patients (pts) with ankylosing spondylitis (AS), fatigue is a common symptom negatively affecting health-related quality of life (HRQoL) and social functioning. Secukinumab (SEC), a fully human anti-IL-17A mAb, rapidly improved signs and symptoms, physical functioning, and HRQoL in pts with AS. ${ }^{1,2}$ Objectives: To assess the long-term effects of SEC on fatigue in TNF inhibitor (TNF)-naïve and TNF inhibitor inadequate responder/intolerant (TNF-IR) AS pts in MEASURE 1 and MEASURE 2.

Methods: 371 and 219 pts were randomized to SEC or placebo (PBO) in MEASURE 1 (10 mg/kg IV followed by 150 or $75 \mathrm{mg} \mathrm{SC}$ ) and MEASURE 2 (150 or $75 \mathrm{mg} \mathrm{SC}$ ), respectively. At Week (Wk) 16, non-responder PBO pts were re-randomized to SEC 150 or $75 \mathrm{mg} \mathrm{SC}$ (in MEASURE 1, PBO pts achieving ASAS20 response at Wk 16 were switched to SEC at Wk 24). Fatigue was measured at baseline (BL) and Wks 4, 8, 12, 16, 24, 52, 104 and 156 using FACIT-F, which assesses fatigue in the previous 7 days using 13 questions graded on a $0-4$ scale (higher scores=less fatigue). An increase from $B L$ in FACIT-F score of $\geq 4$ points (based on MCID) was used to define "response". Approximately $69 \%$ of pts were TNF-naïve and $31 \%$ were TNF-IR across both trials. Analyses were based on the full analysis set and subgroups stratified by prior TNF therapy. Correlations between BL characteristics and improvements in fatigue were investigated using a logistical regression model. Only data from the approved dose (SEC $150 \mathrm{mg}$ ) are presented.

Results: FACIT-F was 24.5-25.6 and 22.6-24.3 at BL across groups in MEASURE 1 and 2, respectively. Improvements in FACIT-F with SEC at Wk 16 were sustained through Wk 156 in MEASURE 1 and Wk 104 in MEASURE 2 (Table). Rapid and sustained fatigue responses were also seen in subgroups stratified by prior TNF use. In the overall population, LS mean changes ( \pm SEM) from BL in FACIT-F scores were significantly greater with SEC vs PBO at WK 16 in both MEASURE $1(7.60 \pm 0.99$ vs $3.34 \pm 1.00, P=0.002)$ and MEASURE $2(8.10 \pm 1.09$ vs $3.27 \pm 1.09$, $P=0.018)$; reductions in fatigue were sustained throughout the entire follow up in both trials (MEASURE 1 Wk 156: 9.81 \pm 0.95 ; MEASURE 2 Wk 104: 9.27 \pm 1.13 ). Similar results were reported in both TNF-naïve and TNF-IR pts. Correlational analyses based on pooled data from both trials did not identify any BL factors that

Table: FACIT-F responders in MEASURE 1 and MEASURE 2 with secukinumab $150 \mathrm{mg}^{\circ}$

\begin{tabular}{|c|c|c|c|c|}
\hline & \multicolumn{4}{|c|}{ Proportion of Patients with FACIT-F Response ${ }^{6}, \%$ (n/m) } \\
\hline & Week 16 & Week 52 & Week 104 & Week 156 \\
\hline \multicolumn{5}{|c|}{ MEASURE $1^{c}$} \\
\hline $\begin{array}{l}\text { Overall } \\
(\mathrm{N}=87)\end{array}$ & $66.7(58 / 87)$ & $73.6(64 / 87)$ & $72.2(57 / 79)$ & $75.6(65 / 86)$ \\
\hline $\begin{array}{l}\text { TNF-naive } \\
(\mathrm{N}=70)\end{array}$ & $70.0(49 / 70)$ & $75.7(53 / 70)$ & $70.3(45 / 64)$ & $74.3(52 / 70)$ \\
\hline $\begin{array}{l}\text { TNF-IR } \\
(\mathrm{N}=17)\end{array}$ & $52.9(9 / 17)$ & $64.7(11 / 17)$ & $80.0(12 / 15)$ & $81.3(13 / 16)$ \\
\hline \multicolumn{5}{|c|}{ MEASURE 2} \\
\hline $\begin{array}{l}\text { Overall } \\
(\mathrm{N}=72)\end{array}$ & $77.6(52 / 67)$ & $80.6(50 / 62)$ & $81.4(48 / 59)$ & - \\
\hline $\begin{array}{l}\text { TNF-naive } \\
(\mathrm{N}=44)\end{array}$ & $86.0(37 / 43)$ & $90.0(36 / 40)$ & $84.6(33 / 39)$ & - \\
\hline $\begin{array}{l}\text { TNF-IR } \\
(\mathrm{N}=28)\end{array}$ & $62.5(15 / 24)$ & $63.6(14 / 22)$ & $75.0(15 / 20)$ & - \\
\hline
\end{tabular}

"Observed data are shown; "Defined as improvement (increase in FACIT-F score) by 24.0 points from BL; "Data shown are from patients who entered the MEASURE 1 long-term extension study at Week 104 (NCT01863732)

$\mathrm{m}$, number of patients with sufficient data for evaluation; N, number of patients randomized to SEC $150 \mathrm{mg}$ $n$, number of responders 